\title{
Inverse P Cygni profiles and their use in astronomical observations
}

\section{Jenny Calahan}

\section{Abstract}

The motivation behind this research project is to better understand protostellar objects and their links to early stellar formation. Specifically, we would like to develop software that will take in an inverse P Cygni line profile of a molecular cloud undergoing infall motion, and give us back the infall rate that is required for that line profile. To do so, we will be editing and using DESPOTIC to take an inverse P Cygni line profile and reverse engineer it in order to get back infall rates such as the infalling velocity, the density, and temperature profiles of the cloud.

\section{Introduction}

In this project, we use what we call line profiles. Line profiles show the intensity of the radiation from a particular transition of a certain molecule as a function of the velocity along the line of sight. A line profile can contain information throughout the cloud because of the Doppler effect. A certain wavelength corresponds to a certain velocity, so we have the ability to see motion of a cloud from front to back. An example of the line of sight and cloud motion is seen in the top part of Figure 1. The dashed line is the line of sight. The specific line profile we use to explore these objects is the inverse P Cygni line profile. 

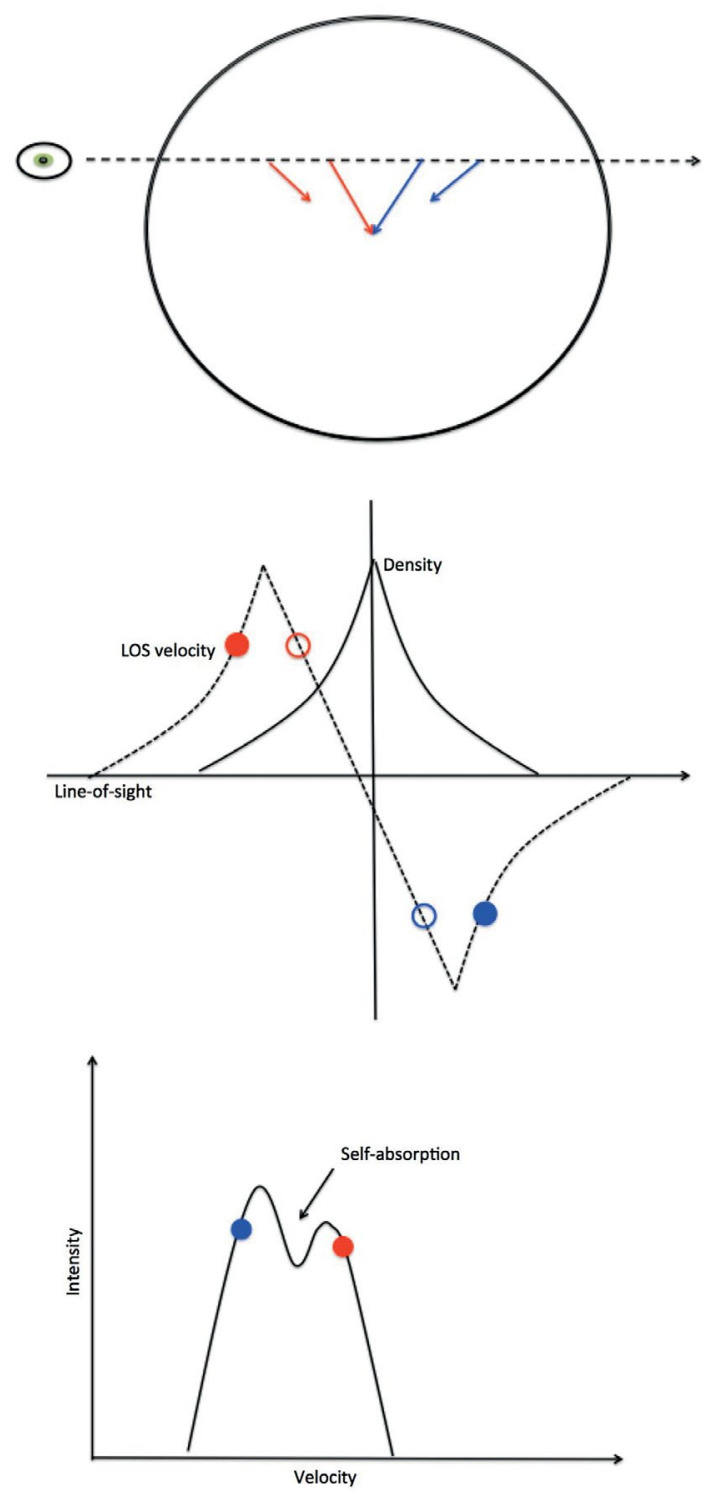

Figure 1: How a line profile describes a molecular cloud. Source: After Smith et al. 2012. 
P Cygni is a luminous blue star that had a unique line profile. While most stars like P Cygni have a line profile that show emission lines (from elements such as hydrogen, helium, and beyond) P Cygni showed absorption lines in the bluer wavelengths and emission lines in redder wavelengths. It became apparent that these now named 'P Cygni' profiles come about when we are observing an object that has a very hot centre, which is surrounded by an outflowing material. ${ }^{1}$

We will be looking at what we call 'inverse P Cygni profiles', which are the exact opposite of what $\mathrm{P}$ Cygni profiles are. The objects we are looking at are collapsing in on themselves, what we call infall motion. A cartoon of how we get a P Cygni profile is shown in Figure 1. As we can see in that figure, we have a core that is collapsing. There is both blueshifted light (material falling towards us) and redshifted light (material falling away from us) represented in the line profile. But, the blueshifted light is more intense than the reshifted light. This isn't because there is more material falling away from us than towards us, but instead it is an effect due to a simple thermodynamic principle.

There are two types of temperatures in play when we talk about radiative transfer, which is the driving force behind these line profiles, and we need to keep both in mind when creating line profiles. There is the kinetic temperature, which is related to the kinetic energy of a molecule and is what we are most familiar with in day-to-day life. Then there is excitation temperature, which is the temperature difference between a molecule in a lower quantum state to an upper quantum state. These temperatures can often be assumed to be equal and, when they are, we call this state local thermodynamic equilibrium, or LTE. If we assume a molecular cloud is in LTE, we can derive the radiative transfer equation using the number density of molecules in the cloud and the kinetic temperature. The radiative transfer equation is the basis behind calculating a line profile, and is described in the next section. 


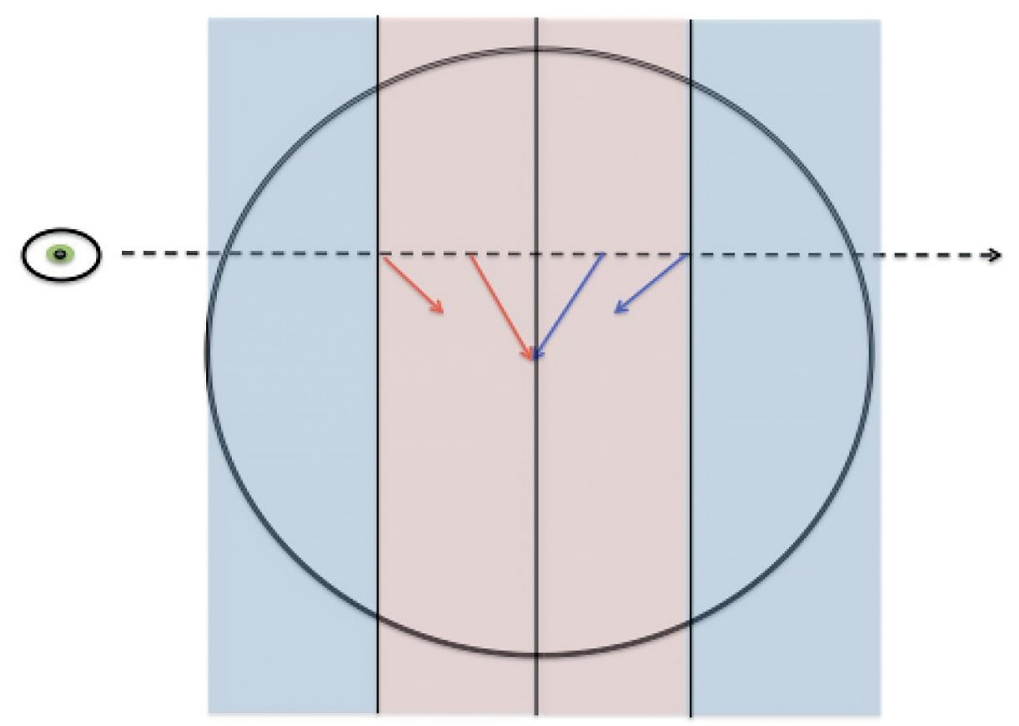

Figure 2: The first part of Figure 1, with added color to describe the kinetic temperature within the cloud. The blue represents hot while the red represents cold. The eye on the left is the observer. Source: After Smith et al. 2012.

To get an inverse P Cygni line profile, we need the temperature in the centre of the cloud to be warmer than the outside of the cloud. In the simplest model of a molecular cloud, we imagine it to be four separate sheets of cloud. This is illustrated in Figure 2. On the redshifted side, there is a cool sheet in front of a hot sheet and continuing through the cloud there is a hot sheet in front of a cool sheet, which is the material that is blueshifted. This is where some thermodynamics comes into play. When we look at the redshifted side, we see cool in front of hot. We also know that when we have a cool gas in front of a hot gas, the cool gas will absorb a lot of the energy from the hot gas. The reverse comes into play on the blueshifted side. There is a hot sheet in front of a cool sheet, so there is not as much absorption as there is on the redshifted side. This is what causes the cloud's line profile to have more intensity on the blueshifted side of the velocity spectra. This is the inverse P Cygni profile, and is also called blue asymmetry. 
However, the kinetic temperature of a molecular cloud can be considered constant, or even cooler in the centre of a molecular cloud. This is because there is no internal heat source within the cloud at the beginning of infall. Only once there is a high enough density in the centre to begin adiabatic heating can we assume that the centre has a higher kinetic temperature than the outside of the cloud. But for a very young protostellar object, we assume that the main source of heat is from background starlight, so the cloud is heated on the outside, but not so much the inside, and the temperature throughout is on the order of $10 \mathrm{~K}$. So, in order to get this inverse P Cygni profile, it is the excitation temperature that makes the difference. In these molecular clouds, we can assume that there is LTE near the centre of the cloud, but not on the outside, so there is a higher excitation temperature in the centre than there is on the outside. It is this excitation temperature that governs the temperature difference that allows us to see an inverse P Cygni profile.

In order to see this line profile, astronomers need to probe the cloud using a certain molecular tracer. The way that we look at a molecular cloud with a radio telescope is we tune the telescope to a certain frequency that corresponds to a transition from a certain excited state of a molecule to a lower energy state. In choosing the molecule to use, we need to be careful. We need a molecule that is relatively abundant, that can be excited at cold temperatures, and has a strong transition, and is somewhere in-between optically thin and optically thick. Optically thick means that a photon emitted in the cloud has much less than a 50 per cent chance of escaping the cloud, but rather will be reabsorbed by the cloud. Optically thin is the exact opposite; a photon emitted has a very high chance of escaping the cloud. A molecule that is completely optically thin would not produce an inverse P Cygni profile, it would be difficult to detect much from the cloud at all. If a cloud is too optically thick, then we do not get the asymmetry that is characteristic in an inverse P Cygni profile.

One might think that molecular hydrogen would be a great molecule to use, because it is by far the most abundant molecule in the universe, and it is sure to be quite abundant in a molecular cloud. But the problem with hydrogen is that at the temperatures that these clouds are at, there are no observed excitations of the molecule at this temperature. Other abundant molecules have different problems. It is possible for them to be too abundant in the cloud, to a point where the cloud becomes optically thick. We need a molecule that not only has active transitions at temperatures around $10 \mathrm{~K}$, but also is abundant to a point where it is between being optically thick and 
optically thin. There are a few special molecules out there that work well. $\mathrm{HCN}, \mathrm{CS}, \mathrm{N}_{2} \mathrm{H}^{+}$, and $\mathrm{HCO}^{+}$are all examples of molecules that still have active transitions at these temperatures, they are all relatively abundant, as well as being marginally optically thick. They all have the ability of creating an inverse P Cygni profile with varying precision.

Looking out for these inverse P Cygni profiles primarily allows us to find and identify molecular clouds that are undergoing infall motion. This is the earliest stage in star formation, and is not fully understood.

\section{DESPOTIC and inverse P Cygni profiles}

We are just starting to understand and create surveys of these prestellar objects. Thanks to the Bolocam Galactic Plane Survey (BGPS), we have discovered over 2,200 massive starless 'clump' candidates. 'Starless clump', being a massive molecular cloud with no stellar activity, meaning there is no internal heat source within the cloud. From this survey, within the 2,200 line profiles, astronomers are searching for infall candidates with inverse P Cygni line profiles (see Figure 3). Once we find these inverse P Cygni profiles we want DESPOTIC, which is software to Derive the Energetics and SPectra of Optically Thick Interstellar Clouds to give us the required infall rates for these line profiles.

1 Brian E. Svoboda et al. 2016. 'The Bolocam Galactic Plane Survey. XIV. Physical Properties of Massive Starless and Star-forming Clumps', The Astrophysical Journal 822(2). 


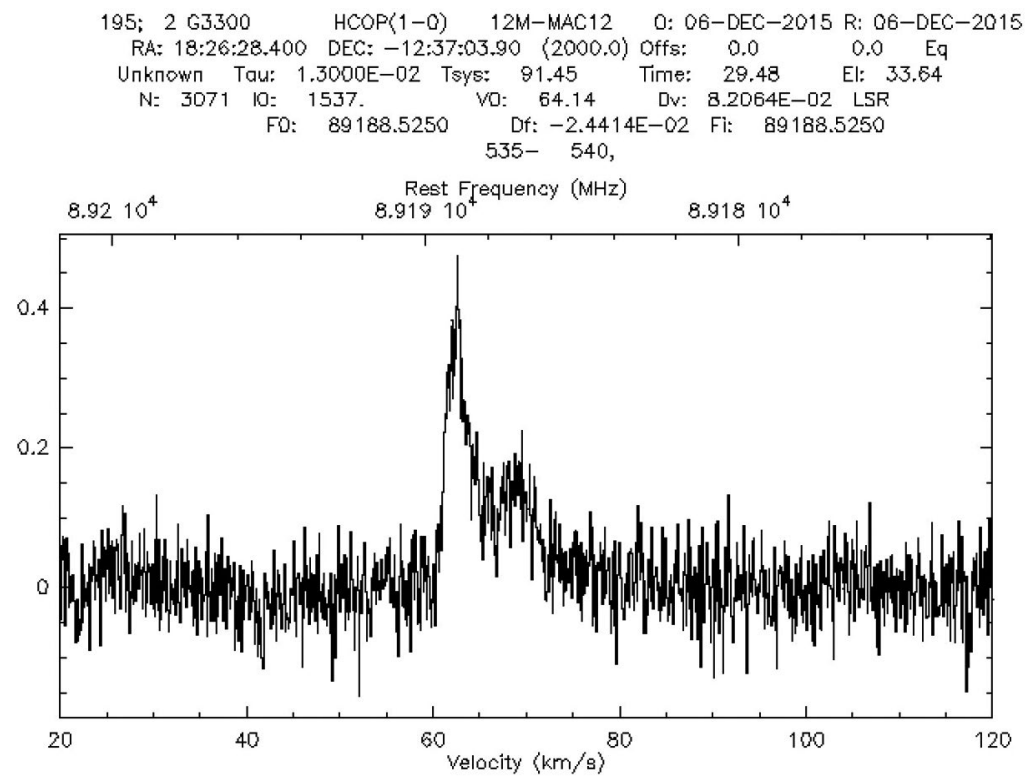

Figure 3. Invers $P$ Cygni line profiles of two infall candidates from the BGPS survey using the chemical tracer $\mathrm{HCO}+1-0$.

Source: Calahan et al. in prep.

In order to accomplish this, we first needed DESPOTIC to probe the cloud using a Gaussian-shaped beam, similar to a radio telescope. In its previous state, the code was set out to create a line profile of a cloud by assuming that radio telescope had an infinitesimally small pencil beam. In reality, radio telescopes have a Gaussian beam. In order to get the code to make a line profile using a Gaussian beam, all we had to do was edit the lineProfLTE.py section of code to integrate a pencil beam over a Gaussian shape, which could have a size as given by the user. We used the original lineProfLTE pencil beam and integrated over the surface of a Gaussian shape. Specifically, we used the following equation to determine the intensity across the cloud:

Equation 1

$I_{\text {avg }}=\int I\left(v, x^{\prime}\right) G\left(\left|x-x^{\prime}\right|\right) d A$ 
Where $I\left(v, x^{\prime}\right)$ was the intensity at velocity $v$ and offset from the center of the cloud $x^{\prime}$ and $G\left(\left|x-x^{\prime}\right|\right)$ is the Gaussian equation with a specified dispersion that is given by the user. If the user did not specify a beam size, then it would assume a pencil beam. We would then integrate $I_{\text {avg }}$ from 0 to 1 , being from the centre of the cloud to the radius. We would end up with an array of $I_{\text {avg }}$ values that corresponded to a $v$ value. This is what became the line profile of the cloud. Using a Gaussian beam in the modelling process makes a big difference in the line profiles. Not only does the shape change from pencil to Gaussian, but also the peak intensity will change, which follows what we see in observations and in the theory. This was a subtle change in the lineProfLTE code, and a simple change to make, but can make a world of difference when modelling molecular cloud line profiles.

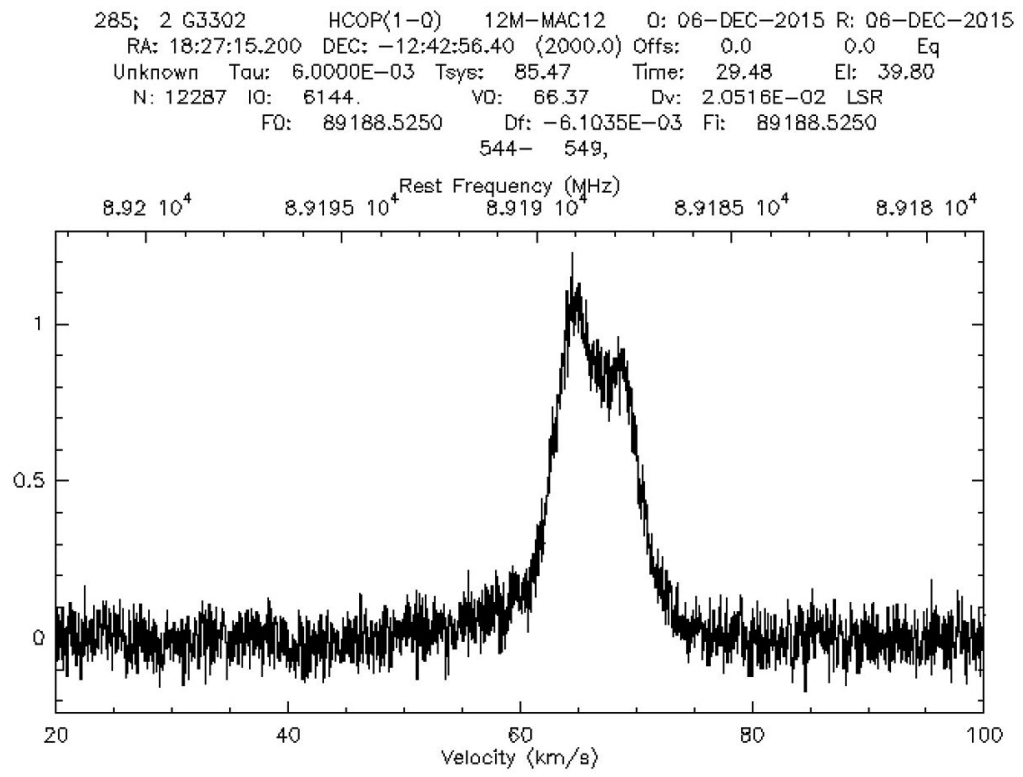

Figure 4: Two line profiles of a molecular cloud with constant parameters except for the beam size.

Source: Calahan et al. in prep. 
Now that we have a more realistic beam for our modelling, we need to run DESPOTIC with certain density, velocity, and temperature profiles as described in Krumholz et al. 2016 and as seen in Equations 2-4. If given the number density of the molecular tracer, the initial temperature $T_{0}$, initial density of the cloud $\rho_{0}$, the radius of the cloud $R$, and the mass infall rate $\dot{M}$, we have enough information to calculate the line profile of a cloud.

\section{Equation 2}

$T(r)=T_{0}\left(\frac{r}{R}\right)^{-0.3}$

\section{Equation 3}

$\rho(r)=\rho_{0}\left(\frac{r}{R}\right)^{-\frac{3}{2}}$

\section{Equation 4}

$v(r)=\frac{\dot{M}}{4 \pi R^{2} \rho_{0}}\left(\frac{r}{R}\right)^{-\frac{1}{2}}$

These conditions of density, temperature, and velocity are derived in Krumholz et al. (2016) and describe material free-falling onto a point mass with a constant mass flux. From these conditions, LineProfLTE numerically integrates the transfer equation, which is derived in Krumholz (2013) as the following: 
$\frac{\mathrm{d} \mathcal{I}_{f}}{\mathrm{~d} x}=n_{s}^{\prime} \frac{g_{u} \mathrm{e}^{-\Theta_{\ell} / t}}{4 \pi Z_{s}(T)}\left[\mathrm{e}^{-\Theta / t}-\tau_{0}\left(1-\mathrm{e}^{-\Theta / t}\right) \mathcal{I}_{f}\right] \phi_{f}$,

where

$\phi_{f}=\frac{1}{\sqrt{2 \pi \sigma_{f}^{2}}} \exp \left[-\frac{\left(f-f_{0}\right)^{2}}{2 \sigma_{f}^{2}}\right]$

$f_{0}=1-\beta u \sin \frac{x}{\sqrt{x^{2}+(d / R)^{2}}}$

$\sigma_{f}=\sqrt{\beta_{s}^{2} t+\beta_{\sigma}^{2} \psi^{2}}$

and we have defined the dimensionless ratios

$\Theta_{\ell}=\frac{E_{\ell}}{k_{B} T(R)} \quad \Theta=\frac{E_{u}-E_{l}}{k_{B} T(R)}$

$\beta=\frac{v(R)}{c} \quad \beta_{s}=\frac{k_{B} T(R)}{\mu_{s} m_{\mathrm{H}} c}$

where

$\phi_{f}=\frac{1}{\sqrt{2 \pi \sigma_{f}^{2}}} \exp \left[-\frac{\left(f-f_{0}\right)^{2}}{2 \sigma_{f}^{2}}\right]$

$f_{0}=1-\beta u \sin \frac{x}{\sqrt{x^{2}+(d / R)^{2}}}$

$\sigma_{f}=\sqrt{\beta_{s}^{2} t+\beta_{\sigma}^{2} \psi^{2}}$

$\frac{\mathrm{d} \mathcal{I}_{f}}{\mathrm{~d} x}=n_{s}^{\prime} \frac{g_{u} \mathrm{e}^{-\Theta_{\ell} / t}}{4 \pi Z_{s}(T)}\left[\mathrm{e}^{-\Theta / t}-\tau_{0}\left(1-\mathrm{e}^{-\Theta / t}\right) \mathcal{I}_{f}\right] \phi_{f}$,

where

$\phi_{f}=\frac{1}{\sqrt{2 \pi \sigma_{f}^{2}}} \exp \left[-\frac{\left(f-f_{0}\right)^{2}}{2 \sigma_{f}^{2}}\right]$

$f_{0}=1-\beta u \sin \frac{x}{\sqrt{x^{2}+(d / R)^{2}}}$

$\sigma_{f}=\sqrt{\beta_{s}^{2} t+\beta_{\sigma}^{2} \psi^{2}}$

and we have defined the dimensionless ratios

$\Theta_{\ell}=\frac{E_{\ell}}{k_{B} T(R)} \quad \Theta=\frac{E_{u}-E_{l}}{k_{B} T(R)}$

$\beta=\frac{v(R)}{c} \quad \beta_{s}=\frac{k_{B} T(R)}{\mu_{s} m_{\mathrm{H}} c}$

Figure 5. Right-hand side of the transfer equation as a function of radius on a logarithmic scale at a velocity of $-10 \mathrm{~km} / \mathrm{s}$.

Source: Krumholz 2013. 
Where $n_{s}^{\prime}=n_{s} / n_{s}(R), t=T / T(R), \psi=\sigma_{N T} / \sigma_{N T}(R), u=v / v(R), f$ is a dimensionless frequency $\frac{v}{\frac{v E}{h}}, g_{u}$ is the degeneracy of the upper states of the molecule, and $Z_{s}(T)$ is the partition function for species $s$ at temperature $\mathrm{T}$.

After defining the transfer equation above using the values that the user gives us, we use the scipy integrate function odeint, and integrate over a range of velocities (that either lineProfLTE creates, or is given to us by the user). What we get out of that is an array of intensity values that we can plot verse the range of velocities in order to plot the line profile.

We made some changes to the lineprofLTE code in attempts to make it more precise. First, we adjusted the radius limits that we gave odeint, originally we just gave it the outer bounds, from the back of the cloud to the front of the cloud. We then added a limit that was the radius at which the line of sight velocity was, to make sure the integrator hit that key point. We also added points around it. A visual representation of this is seen in Figure 5 where we graph the right-hand side of the transfer equation as a function of radius at a given velocity. The radius at the line of sight is the point at which the transfer equation peaks and it varies depending on the velocity you are exploring. Solely adding those limits was not enough, because in linear space, that jump in the transfer equation is like a delta function, especially when you go up to higher velocities, it is very hard for the odeint function to find. So, we made a new definition for the right-hand side of the transfer equation. We told lineProfLTE to integrate over a log scale where we wrote the normalised radius in terms of a log scale. We did this because it was easy to for us to see the point in which the transfer function peaked, and so could odeint. We had to write a new version of the transfer equation. We wanted the following:

$\frac{d I}{d \log (r)}=\frac{d I}{d s} * \frac{d s}{d \log (r)}$

Where $\frac{d I}{d s}$ was from the normal transfer equation and $\frac{d s}{\operatorname{dlog}(r)}$ depended on the location of the beam. With this change, the resulting line profile was much more realistic, and we had made lineProfLTE more flexible for other astronomers using the program.

With this code, we will be able to create a line profile with known density, temperature, and velocity dispersion. We can then add some noise to that line profile and use a fitting program from scipy on that noisy line profile. Using 
that fitting program and the line profile with known features, we can figure out ways to start with an observed profile and derive the density, temperature, and velocity profiles of the cloud. We will have the ability to take an infall candidate from the BGPS and use DESPOTIC to then vary the parameters until a fit is found.

\section{Future applications}

Despotic is a very exciting tool that is making huge strides in the modelling and overall understanding of protostellar objects and stellar formation. With this project in particular, we have improved the ability to model a molecular cloud undergoing infall motion. Growing on that, it would be incredibly helpful if we were able to take an observed line profile and determine the density, velocity, and temperature profile of that cloud. The second part of the project has taken the first steps in being able to do just that.

Within that large Bolocam Galactic Plane Survey of 2,200 starless candidates, we at the University of Arizona are combing through and blindly searching for infall candidates. We do this by creating a line profiles for the best starless clump candidates and when we find an object that has an inverse P Cygni profile, we call that a good candidate for infall motion, which, as discussed, is the precursor for stellar formation. After identifying and integrating over around 100 objects, we found 6 objects that were good candidates for infall. For further confirmation of infall motion, it is necessary to use bigger radio telescopes with higher resolution in order to get a more detailed view with the cloud. But, in the meantime, there is not much information we can get from that profile, just that it is a good candidate for infall and we can find ways to quantitatively express the degree of blue asymmetry. If we were able to determine such things as the density, temperature, and/or velocity profile of the cloud it would unearth extremely valuable information, which would be incredibly useful and insightful. Perhaps we can determine how good of an infall candidate it is, and then we do not have to waste time on a highly sought after research telescope. We could compare and contrast these parameters with the mass of the cloud, the distance from us, and the size of the cloud, which is information we have from BGPS. The possibilities seem endless if we could get that information just from a cloud's line profile. There is just still so much we 
do not understand about stellar formation, and being able to answer a few of these questions will help us understand these objects that underlie everything from planetary to galactic evolution.

\section{References}

American Association of Variable Star Observers. 'P Cygni', last modified 13 April 2010, accessed 23 October 2016, www.aavso.org/vsots_pcyg.

Calahan, Jenny et al. (forthcoming). 'Searching for Infall Candidates of High Mass Molecular Clumps', BSci(Hons) thesis, University of Arizona.

Krumholz, Mark R. 2013. 'DESPOTIC - a new software library to Derive the Energetics and SPectra of Optically Thick Interstellar Clouds'. Monthly Notices of the Royal Astronomical Society 437: 1662-1680. doi.org/10.1093/mnras/stt2000.

Krumholz, Mark R., Andrew T. Myers, Richard I. Klein, and Christopher F. McKee. 2016. 'What physics determines the peak of the IMF? Insights from the structure of cores in radiation-magnetohydrodynamic simulations', Monthly Notices of the Royal Astronomical Society 460: 3272 3283. doi.org/10.1093/mnras/stw1236.

Smith, Rowan J., Rahul Shetty, Amelia M. Stulz, and Ralf S. Klessen. 2012. 'Line Profiles Of Cores Within Clusters. I. The Anatomy Of A Filament', The Astrophysical Journal 750(1). doi.org/10.1088/0004-637X/750/1/64.

Svoboda, Brian E., Yancy L. Shirley, Cara Battersby, Erik W. Rosolowsky, Adam G. Ginsburg, Timothy P. Ellsworth-Bowers, Michele R. Pestalozzi, Miranda K. Dunham, Neal J. Evans II, John Bally, and Jason Glenn. 2016. 'The Bolocam Galactic Plane Survey. XIV. Physical Properties of Massive Starless and Star-forming Clumps', The Astrophysical Journal 822(2). doi.org/10.3847/0004-637X/822/2/59. 
This text is taken from Merici, Volume 2, 2016, edited by Matthew Rogers, published 2017 by ANU eView, The Australian National University,

Canberra, Australia. 